(C) 2000 International Press

Adv. Theor. Math. Phys. 3 (2000) 615-626

\title{
Graded KMS-Functionals and the Breakdown of
}

\section{Supersymmetry}

Detlev Buchholz and Roberto Longo ${ }^{1}$

Institut für Theoretische Physik, Universität Göttingen, Bunsenstraße 9, D-37073 Göttingen, Germany buchholz@theorie.physik.uni-goettingen.de

Università di Roma "Tor Vergata"

Via della Ricerca Scientifica, I-00133 Roma, Italy

longo@mat.uniroma2.it

\begin{abstract}
It is shown that the modulus of any graded or, more generally, twisted KMS-functional of a $\mathrm{C}^{*}$-dynamical system is proportional to an ordinary KMS-state and the twist is weakly inner in the corresponding GNS-representation. If the functional is invariant under the adjoint action of some asymptotically abelian family of automorphisms, then the twist is trivial.
\end{abstract}

\footnotetext{
${ }^{1}$ Supported in part by GNAFA and MURST.

e-print archive: http://xxx.lanl.gov/hep-th/9905102
} 
As a consequence, such functionals do not exist for supersymmetric $\mathrm{C}^{*}$-dynamical systems. This is in contrast with the situation in compact spaces where super KMS-functionals occur as super-Gibbs functionals.

\section{Introduction}

Graded KMS-functionals play a prominent role, both, in physics and in mathematics. In physics they are used as a tool in the construction of supersymmetric quantum field theories in a thermal background $[5,6]$. In mathematics they appear in non-commutative geometry, notably in the context of Connes' cyclic cohomology $[7,8]$ and of the Witten index [11].

It is the aim of the present article to exhibit in the general setting of $\mathrm{C}^{*}$-dynamical systems some elementary properties of graded (or, more generally, twisted) KMS-functionals which seem to have escaped observation so far.

The first part of our analysis is complementary to the work of Stoytchev [9], who proved that any normal, faithful and symmetric functional on a von Neumann algebra is a graded KMS-functional with respect to the action of some involution and some canonically associated (modular) automorphism group. We will show here in the $\mathrm{C}^{*}$-algebraic setting that the modulus of any twisted KMS functional is a multiple of an ordinary KMS-state and the twist is weakly inner in the corresponding GNS-representation.

This structure is familiar from numerous concrete examples of supersymmetric dynamical systems in compact space. But, as is shown in the second part of our article, it disappears if one adds the assumption that the twisted functional is invariant under the action of some asymptotically abelian family of automorphisms, which is typical of infinite systems (thermodynamic limit). Namely, the twist becomes trivial in this case and the functional satisfies the ordinary KMS condition. It is a simple consequence of this result that such functionals cannot be accommodated in supersymmetric theories.

These results provide further evidence to the effect that thermal 
systems can be supersymmetric only in compact space. For if the spacetime admits a group of symmetries shifting points spacelike to infinity, then supersymmetric thermal states can never be homogeneous with respect to that action.

That supersymmetry is extremely vulnerable to thermal effects in infinite systems was first pointed out in a model independent setting in [1], where it was shown that supersymmetry is necessarily broken in all spatially homogeneous KMS states. These results were carried over to a $\mathrm{C}^{*}$-algebraic setting and generalized in [2].

The present results show that graded KMS functionals, which are frequently taken as building blocks in the construction of supersymmetric models, would not only break supersymmetry if one proceeds to the thermodynamic limit [1], they simply cease to exist. Some implications of this observation for the study of infinite dynamical systems are discussed in the conclusions.

\section{Twisted KMS functionals}

Let $\mathfrak{A}$ be a unital $\mathrm{C}^{*}$-algebra, $\alpha$ a one-parameter automorphism group acting on $\mathfrak{A}$, and $\gamma$ an automorphism of $\mathfrak{A}$. Thus $(\mathfrak{A}, \alpha)$ is a (not necessarily continuous) $\mathrm{C}^{*}$-dynamical system and $\gamma$ defines a twist on $\mathfrak{A}$. We are primarily interested in the case $\gamma^{2}=$ id, i.e., where $\gamma$ is a $\mathbb{Z}_{2}$-grading on $\mathfrak{A}$, but it will be useful not to assume this from the outset.

We shall say that a bounded, linear (not necessarily positive) functional $\varphi$ on $\mathfrak{A}$ is a $\gamma$-twisted KMS functional (or simply a twisted KMS functional) if, for any given $a, b \in \mathfrak{A}$, there exists a complex function $F_{a, b} \in A(S)$ with

$$
\begin{aligned}
F_{a, b}(t) & =\varphi\left(\alpha_{t}(a) b\right) \\
F_{a, b}(t+i) & =\varphi\left(\gamma(b) \alpha_{t}(a)\right), \quad t \in \mathbb{R} .
\end{aligned}
$$

Here $A(S)$ is the set of bounded continuous functions on the strip $S \equiv\{0 \leq \Im z \leq 1\}$ which are analytic in the interior of $S$. In physics the width of the strip $S$ has the meaning of inverse temperature which we have normalized here to 1 for convenience. 
The same argument as for ordinary ( $\gamma=\mathrm{id}) \mathrm{KMS}$ states implies that a $\gamma$-twisted KMS functional $\varphi$ is $\alpha$-invariant: Since $F_{a, 1}(t)=F_{a, 1}(t+i)$ the function $F_{a, 1}$ extends to a bounded entire function and therefore is constant. Setting $a=1$ in (1), we also see that $\varphi$ is $\gamma$-invariant.

Given a $\gamma$-twisted KMS functional $\varphi$, we consider its modulus $\omega \equiv$ $|\varphi|$ which is obtained by extending $\varphi$ to the second dual of $\mathfrak{A}$ and subsequent polar decomposition. Equivalently, $\omega$ may be characterized $[4$, Sec. 12.2.9] as the unique positive linear functional on $\mathfrak{A}$ which satisfies $\|\omega\|=\|\varphi\|$ and

$$
|\varphi(a)|^{2} \leq\|\varphi\| \omega\left(a^{*} a\right), \quad a \in \mathfrak{A} .
$$

From the latter characterization one sees that there holds $|\varphi \cdot \beta|=\omega \cdot \beta$ for any automorphism $\beta \in$ Aut $\mathfrak{A}$. Hence if $\beta$ preserves $\varphi$, i.e., $\varphi \cdot \beta=\varphi$, then it also preserves $\omega$.

Proceeding to the GNS-representation $\{\pi, \mathcal{H}, \Omega\}$ of $\mathfrak{A}$ induced by $\omega$, one can implement any automorphism $\beta$ which preserves $\varphi$ by a unitary operator $U_{\beta}$ on $\mathcal{H}$. It is determined by

$$
U_{\beta} a \Omega \equiv \beta(a) \Omega, \quad a \in \mathfrak{A} .
$$

We may assume that $\pi$ is $1-1$ (replacing $\mathfrak{A}$ by $\mathfrak{A} / \operatorname{ker} \pi$ if necessary), therefore, here and in the subsequent discussion, we identify $\mathfrak{A}$ with its image $\pi(\mathfrak{A})$ under the homomorphism $\pi$ in order to simplify the notation. With this convention, $\beta$ extends to an automorphism $\widetilde{\beta}$ of the weak closure $\mathfrak{M}=\mathfrak{A}^{\prime \prime}$ given by $\widetilde{\beta}=\operatorname{Ad} U_{\beta}$.

As is well known (and can be seen from (2)) the functional $\varphi$ can be represented in the form (polar decomposition)

$$
\varphi=(u \cdot \Omega, \Omega) .
$$

Here $u \in \mathfrak{M}$ is a partial isometry which is uniquely fixed by the condition that $u u^{*}$ is the support projection of $\omega$, i.e., the smallest projection $p \in \mathfrak{M}$ for which $p \Omega=\Omega$.

We consider in the following the canonical extensions of $\omega, \varphi$ to $\mathfrak{M}$, which are given by $\widetilde{\omega} \equiv(\cdot \Omega, \Omega)$ and $\widetilde{\varphi} \equiv(u \cdot \Omega, \Omega)$, respectively. The following result can be established by standard arguments, cf. [10].

Lemma 1. $\tilde{\varphi}$ is a $\tilde{\gamma}$-twisted KMS functional of $(\mathfrak{M}, \tilde{\alpha})$. 
Proof. If $x, y \in \mathfrak{M}$, there exist by Kaplansky's density theorem bounded nets $a_{i} \in \mathfrak{A}$ and $b_{j} \in \mathfrak{A}$ such that $a_{i} \rightarrow x$ and $b_{j} \rightarrow y^{*_{-}}$ strongly. The corresponding family of functions $\left\{t \mapsto \varphi\left(\alpha_{t}\left(a_{i}\right) b_{j}\right)\right\}_{i, j}$ converges to $t \mapsto \widetilde{\varphi}\left(\widetilde{\alpha}_{t}(x) y\right)$ uniformly on $\mathbb{R}$, as can be seen from the estimate

$$
\begin{aligned}
& \left|\varphi\left(\alpha_{t}\left(a_{i}\right) b_{j}\right)-\widetilde{\varphi}\left(\widetilde{\alpha}_{t}(x) y\right)\right| \\
& \leq\left\|\left(a_{i}-x\right)^{*} u^{*} \Omega\right\|\left\|b_{j} \Omega\right\|+\left\|\left(b_{j}-y\right) \Omega\right\|\left\|x^{*} u^{*} \Omega\right\| .
\end{aligned}
$$

Here the $\widetilde{\alpha}$-invariance of $\widetilde{\varphi}, \widetilde{\omega}$ and the polar decomposition of $\widetilde{\varphi}$ have been used. In a similar manner one sees that the family $\left\{t \mapsto \varphi\left(\gamma\left(b_{j}\right) \alpha_{t}\left(a_{i}\right)\right)\right\}_{i, j}$ converges to $t \mapsto \widetilde{\varphi}\left(\widetilde{\gamma}(y) \widetilde{\alpha}_{t}(x)\right)$ uniformly on $\mathbb{R}$. This implies, since the maximum modulus principle holds on $A(S)$ according to the Three-Line-Theorem, that the family $\left\{F_{a_{i}, b_{j}}\right\}_{i, j}$ converges uniformly on the strip $S$ to some function $\widetilde{F}_{x, y}$ which also belongs to $A(S)$. Moreover, $\widetilde{F}_{x, y}(t)=\widetilde{\varphi}\left(\widetilde{\alpha}_{t}(x) y\right)$ and $\widetilde{F}_{x, y}(t+i)=\widetilde{\varphi}\left(\widetilde{\gamma}(y) \widetilde{\alpha}_{t}(x)\right)$.

Lemma 2. Let $\omega$ be a $\gamma$-twisted KMS functional of $(\mathfrak{A}, \alpha)$. If $\omega$ is positive, then $\tilde{\gamma}=\mathrm{id}$ and $\widetilde{\omega}$ is an ordinary positive KMS functional of $(\mathfrak{M}, \widetilde{\alpha})$.

Proof. As $\omega$ is positive it coincides with its modulus and the associated partial isometry $u \in \mathfrak{M}$ satisfies $u^{*} \Omega=\Omega$. We shall show next that $\Omega$ is separating for $\mathfrak{M}$. Let $U(t)=e^{i H t}$ be the one-parameter unitary group implementing $\widetilde{\alpha}_{t}$, cf. relation (3), and let $V$ be the unitary operator implementing $\widetilde{\gamma}$. According to Lemma 1 , the functional $\widetilde{\omega}$ is a $\widetilde{\gamma}$-twisted positive KMS functional for $(\mathfrak{M}, \widetilde{\alpha})$. Now if $x \in \mathfrak{M}$ is such that $x \Omega=0$, then also $\widetilde{\alpha}_{t}(x) \Omega=0$, hence, making use of formula (1), we have for all $y \in \mathfrak{M}$

$$
\begin{aligned}
& x \Omega=0 \Rightarrow \widetilde{\omega}\left(\widetilde{\gamma}(y) \widetilde{\alpha}_{t}(x)\right)=\left(\widetilde{\gamma}(y) \widetilde{\alpha}_{t}(x) \Omega, \Omega\right)=0 \\
& \quad \Rightarrow \widetilde{\omega}(x y)=0 \Rightarrow\left(y \Omega, x^{*} \Omega\right)=0 \Rightarrow x^{*} \Omega=0 .
\end{aligned}
$$

Thus $x \Omega=0 \Rightarrow z x \Omega=0 \Rightarrow x^{*} z^{*} \Omega=0$ for all $z \in \mathfrak{M}$, hence $x=0$. As an immediate consequence of this observation we have $u^{*}=1$. It remains to show that $\widetilde{\gamma}=\mathrm{id}$.

Let $\Delta^{i t}$ be the one-parameter modular group associated with $\mathfrak{M}$ and $\Omega$. As $\Omega$ is $U(s)$-invariant and $\widetilde{\alpha}_{s}(\mathfrak{M})=\mathfrak{M}$, the unitary groups 
$\Delta^{i t}$ and $U(s)$ commute. Thus there exists a dense subalgebra $\mathfrak{M}_{0} \subset \mathfrak{M}$ such that $\mathfrak{M}_{0} \Omega$ is a core both for $\Delta$ and $e^{H}$. By the KMS property for the modular group we have

$$
\left(x^{*} \Omega, y^{*} \Omega\right)=\left(y x^{*} \Omega, \Omega\right)=\left(x^{*} \Delta y \Omega, \Omega\right)=(\Delta y \Omega, x \Omega), \quad x, y \in \mathfrak{M}_{0} .
$$

On the other hand, by the $\gamma$-twisted KMS condition, we have

$$
\begin{array}{r}
\left(x^{*} \Omega, y^{*} \Omega\right)=\left(y x^{*} \Omega, \Omega\right)=\left(\widetilde{\gamma}\left(x^{*}\right) e^{H} y \Omega, \Omega\right)=\left(e^{H} y \Omega, \widetilde{\gamma}(x) \Omega\right) \\
=\left(e^{H} y \Omega, V x \Omega\right)=\left(V^{*} e^{H} y \Omega, x \Omega\right), \quad x, y \in \mathfrak{M}_{0} .
\end{array}
$$

Hence $\Delta=V^{*} e^{H}$ and, by the uniqueness of the polar decomposition for closed linear operators, $\Delta=e^{H}$ and $V=1$, i.e., $\widetilde{\gamma}=$ id.

Lemma 3. Let $\varphi$ be a $\gamma$-twisted KMS functional of $(\mathfrak{A}, \alpha)$ and let $\varphi=(u \cdot \Omega, \Omega)$ be its polar decomposition. Then $u$ is unitary and $\Omega$ is separating for $\mathfrak{M}$.

Proof. We begin by noting that $u$ is left fixed both by $\widetilde{\gamma}$ and $\widetilde{\alpha}$ because of the invariance of $\widetilde{\varphi}$ and $\widetilde{\omega}$ under the adjoint action of these automorphisms and the uniqueness of the polar decomposition. Now, for $\widetilde{\alpha}$-analytic elements $x \in \mathfrak{M}$ the twisted KMS condition for $\widetilde{\varphi}$ can be expressed as

$$
\widetilde{\omega}\left(u \widetilde{\alpha}_{i}(x) y\right)=\widetilde{\omega}(u \widetilde{\gamma}(y) x) .
$$

Replacing $x$ with $u^{*} x$ and using the $\widetilde{\alpha}$-invariance of $u$ as well as the fact that $u u^{*}$ is the support projection of $\widetilde{\omega}$, we can proceed to

$$
\widetilde{\omega}\left(\widetilde{\alpha}_{i}(x) y\right)=\widetilde{\omega}\left(u \widetilde{\gamma}(y) u^{*} x\right) .
$$

Setting $x=u$ and $y=u^{*}$ in this formula we get, taking into account that $\widetilde{\gamma}\left(u^{*}\right)=u^{*}$,

$$
\widetilde{\omega}(1)=\widetilde{\omega}\left(u u^{*}\right)=\widetilde{\omega}\left(u u^{*} u^{*} u\right)=\widetilde{\omega}\left(u^{*} u\right),
$$

hence $u^{*} u \Omega=u u^{*} \Omega=\Omega$ by the limit case of the Schwartz inequality.

We shall show now that $\Omega$ is separating for $\mathfrak{M}$, and this will follow as above by showing that $x \Omega=0$ for $x \in \mathfrak{M}$ implies $x^{*} \Omega=0$. So let 
$x \in \mathfrak{M}$ satisfy $x \Omega=0$. Then $\widetilde{\varphi}\left(y \widetilde{\alpha}_{t}(x)\right)=\left(u y \widetilde{\alpha}_{t}(x) \Omega, \Omega\right)=0$ for all $y \in \mathfrak{M}$. Hence by equation (1) we have $(u \widetilde{\gamma}(x) y \Omega, \Omega)=\widetilde{\varphi}(\widetilde{\gamma}(x) y)=0$ and this implies $\widetilde{\gamma}\left(x^{*}\right) u^{*} \Omega=0$ or equivalently, since $\widetilde{\gamma}\left(u^{*}\right)=u^{*}$, that $x^{*} u^{*} \Omega=0$. As $u u^{*}$ is the support projection of $\widetilde{\omega}$ we conclude that $x^{*} u^{*}=x^{*} u^{*} u u^{*}=0$ and consequently $x^{*} u^{*} u=0$. But $u^{*} u \Omega=\Omega$, so it follows that $x^{*} \Omega=0$, i.e., $\Omega$ is separating. Hence $u^{*} u=u u^{*}=1$.

We mention as an aside that the above result allows one to disintegrate a twisted KMS functional into factorial twisted KMS functionals.

Proposition 4. Let $\varphi$ be a $\gamma$-twisted KMS functional of $(\mathfrak{A}, \alpha)$ and let $\varphi=(u \cdot \Omega, \Omega)$ be its polar decomposition. Then $\widetilde{\gamma}$ is inner on $\mathfrak{M}$, indeed $\widetilde{\gamma}=\operatorname{Ad} u^{*}$, and $\widetilde{\omega}$ is an ordinary positive KMS functional for $(\mathfrak{M}, \widetilde{\alpha})$.

Proof. Since $u$ is unitary we have $\tilde{\theta} \equiv \operatorname{Ad} u \cdot \widetilde{\gamma} \in$ Aut $\mathfrak{M}$, so formula (10) shows that $\widetilde{\omega}$ is a $\widetilde{\theta}$-twisted KMS functional of $(\mathfrak{M}, \widetilde{\alpha})$. (We recall that this formula amounts to relation (1) for $\widetilde{\alpha}$-entire elements $x \in \mathfrak{M}$; that it entails relation (1) for all elements of $\mathfrak{M}$ can be shown by similar arguments as in the proof of Lemma 1.) As $\widetilde{\omega}$ is positive, we conclude from Lemma 2 that $\widetilde{\theta}=$ id. Hence $\widetilde{\omega}$ is an ordinary positive KMS functional and $\widetilde{\gamma}=\operatorname{Ad} u^{*}$.

Corollary 5. If $\beta \in$ Aut $\mathfrak{A}$ preserves the $\gamma$-twisted $K M S$ functional $\varphi$ of $(\mathfrak{A}, \alpha)$, then $\widetilde{\beta}$ commutes with $\widetilde{\alpha}$ and $\widetilde{\gamma}$.

Proof. Since $\widetilde{\beta}(u)=u$ by the uniqueness of the polar decomposition of $\varphi$, the commutativity of $\widetilde{\beta}$ and $\widetilde{\gamma}$ follows from the preceding result. As $\widetilde{\alpha}$ is the modular group associated with $(\mathfrak{M}, \Omega)$, it is also clear that $\widetilde{\beta}$ commutes with $\widetilde{\alpha}$.

\section{$3 \quad$ Twisted asymptotic abelianess}

We shall specialize now to the class of $\mathrm{C}^{*}$-dynamical systems $(\mathfrak{A}, \alpha)$ for which there exists a family of automorphisms acting on $\mathfrak{A}$ in a (twisted) 
asymptotically abelian manner. This situation prevails in physics if one deals with infinite systems (thermodynamic limit). As a matter of fact, the dynamics $\alpha$ itself is asymptotically abelian in generic cases.

Given a $\gamma$-twisted KMS functional $\varphi$ of $(\mathfrak{A}, \alpha)$, we shall say that a sequence of automorphisms $\beta_{n} \in$ Aut $\mathfrak{A}$ is $\varphi$-asymptotically abelian if $\varphi \cdot \beta_{n}=\varphi$ and

$$
\lim _{n} \varphi\left(c\left[a, \beta_{n}(b)\right]\right)=0 \quad \text { for all } a, b, c \in \mathfrak{A} .
$$

Here the twisted commutator is defined by $[a, b] \equiv a b-\gamma(b) a$.

Lemma 6. Let $\varphi$ be a $\gamma$-twisted $K M S$ functional of $(\mathfrak{A}, \alpha)$. If there exists a $\varphi$-asymptotically abelian sequence $\beta_{n} \in$ Aut $\mathfrak{A}$, then $\left[x, \widetilde{\beta}_{n}(y)\right] \rightarrow$ 0 weakly for all $x, y \in \mathfrak{M}$.

Proof. Since $\left(\left[a, \beta_{n}(b)\right] \Omega, c^{*} u^{*} \Omega\right)=\varphi\left(c\left[a, \beta_{n}(b)\right]\right)$ and the set of vectors $c^{*} u^{*} \Omega, c \in \mathfrak{A}$, is dense in $\mathcal{H}$ (recall that $u$ is a unitary in $\mathfrak{M}=\mathfrak{A}^{\prime \prime}$ ), it follows from condition (12) that the (bounded) sequence $\left[a, \beta_{n}(b)\right] \Omega$ converges weakly to 0 . Thus $\left[a, \beta_{n}(b)\right] \rightarrow 0$ weakly because all weak limit points of $\left[a, \beta_{n}(b)\right]$ are elements of $\mathfrak{M}$ and $\Omega$ is separating for $\mathfrak{M}$ by Lemma 3 .

As the unit ball $\mathfrak{A}_{1}$ of $\mathfrak{A}$ is ${ }^{*}$-strongly dense in the unit ball $\mathfrak{M}_{1}$ of $\mathfrak{M}$ by Kaplansky's density theorem, given $x, y \in \mathfrak{M}_{1}$ and $\varepsilon>0$, there exist $a, b \in \mathfrak{A}_{1}$ with

$$
\|x \Omega-a \Omega\|,\left\|x^{*} \Omega-a^{*} \Omega\right\|,\|y \Omega-b \Omega\|,\left\|y^{*} \Omega-b^{*} \Omega\right\|<\varepsilon .
$$

Now for any fixed $z^{\prime} \in \mathfrak{M}_{1}^{\prime}$ we have

$$
\begin{aligned}
& \left|\left(\left[x, \widetilde{\beta}_{n}(y)\right] \Omega, z^{\prime} \Omega\right)-\left(\left[a, \widetilde{\beta}_{n}(b)\right] \Omega, z^{\prime} \Omega\right)\right| \\
& \leq\left|\left(\left(x \widetilde{\beta}_{n}(y)-a \widetilde{\beta}_{n}(b)\right) \Omega, z^{\prime} \Omega\right)\right| \\
& \quad+\left|\left(\left(\widetilde{\gamma}\left(\widetilde{\beta}_{n}(y)\right) x-\widetilde{\gamma}\left(\widetilde{\beta}_{n}(b)\right) a\right) \Omega, z^{\prime} \Omega\right)\right| .
\end{aligned}
$$

The first term in the right hand side of the above inequality can be estimated by

$$
\begin{aligned}
& \left|\left(\left(x \widetilde{\beta}_{n}(y)-a \widetilde{\beta}_{n}(b)\right) \Omega, z^{\prime} \Omega\right)\right| \\
& \leq\left|\left((x-a) \widetilde{\beta}_{n}(y) \Omega, z^{\prime} \Omega\right)\right|+\left|\left(a \widetilde{\beta}_{n}(y-b) \Omega, z^{\prime} \Omega\right)\right| \\
& \leq\left|\left(\widetilde{\beta}_{n}(y) \Omega, z^{\prime}(x-a)^{*} \Omega\right)\right|+\left|\left(\widetilde{\beta}_{n}(y-b) \Omega, z^{\prime} a^{*} \Omega\right)\right| \leq 2\|\Omega\|^{2} \varepsilon,
\end{aligned}
$$


where, in the last step, we used the fact that $\widetilde{\beta}_{n}$ preserves $\omega$. Similarly, the second term on the right hand side of (14) is bounded by $2\|\Omega\|^{2} \varepsilon$.

Since $\varepsilon$ is arbitrary, this entails $\left(\left[x, \widetilde{\beta}_{n}(y)\right] \Omega, z^{\prime} \Omega\right) \rightarrow 0$, hence, as the set of vectors $\mathfrak{M}^{\prime} \Omega$ is dense in $\mathcal{H}$ and $\Omega$ is separating for $\mathfrak{M}$, we conclude that $\left[x, \widetilde{\beta}_{n}(y)\right] \rightarrow 0$ weakly for all $x, y \in \mathfrak{M}$.

Proposition 7. Let $\varphi$ be a $\gamma$-twisted KMS functional of $(\mathfrak{A}, \alpha)$. If there exists a $\varphi$-asymptotically abelian sequence $\beta_{n} \in$ Aut $\mathfrak{A}$, then $\widetilde{\gamma}=\mathrm{id}$ and $\widetilde{\varphi}$ is an ordinary $K M S$ functional of $(\mathfrak{M}, \widetilde{\alpha})$.

Proof. As noticed above, there holds $\widetilde{\beta}_{n}(u)=\widetilde{\gamma}(u)=u$ by the uniqueness of the polar decomposition of $\varphi$. Thus, by the preceding lemma,

$$
\begin{aligned}
x u-u x=x \widetilde{\beta}_{n}(u)-\widetilde{\beta}_{n}(u) x & =x \widetilde{\beta}_{n}(u)-\widetilde{\gamma}\left(\widetilde{\beta}_{n}(u)\right) x \\
& =\left[x, \widetilde{\beta}_{n}(u)\right] \rightarrow 0
\end{aligned}
$$

weakly for all $x \in \mathfrak{M}$. So $u$ belongs to the center of $\mathfrak{M}$ and, as $\widetilde{\gamma}=\operatorname{Ad} u^{*}$ by Proposition 4, $\widetilde{\gamma}$ is trivial.

Let us now assume that $\widetilde{\gamma}^{2}=$ id. Note that this is always the case if the $\gamma$-twisted KMS functional $\varphi$ is selfadjoint, i.e., $\varphi\left(a^{*}\right)=\overline{\varphi(a)}$, $a \in \mathfrak{A}$. For then

$$
\widetilde{\omega}(u a)=\varphi(a)=\overline{\varphi\left(a^{*}\right)}=\overline{\widetilde{\omega}\left(u a^{*}\right)}=\widetilde{\omega}\left(a u^{*}\right)=\widetilde{\omega}\left(u^{*} a\right), \quad a \in \mathfrak{A},
$$

where in the last equality we made use of the fact that $\widetilde{\omega}$ is a KMS functional and $\widetilde{\alpha}_{t}\left(u^{*}\right)=u^{*}$. Thus $u=u^{*}$ since $\widetilde{\omega}$ is faithful, hence $\widetilde{\gamma}^{2}=\operatorname{Ad} u^{* 2}=$ id. A $\gamma$-twisted KMS functional is called graded KMS functional if $\widetilde{\gamma}$ has the latter property.

Given a graded KMS-functional $\varphi$ of $(\mathfrak{A}, \alpha)$, let $\mathfrak{M}_{\text {an }} \subset \mathfrak{M}$ be the weakly dense algebra of $\widetilde{\alpha}$-analytic elements and let

$$
\widetilde{\delta}_{0} \equiv-\left.i \frac{\mathrm{d}}{\mathrm{d} t} \widetilde{\alpha}_{t}\right|_{t=0}
$$

be the generator of $\widetilde{\alpha}$, which has $\mathfrak{M}_{\mathrm{an}}$ as a core. The dynamical system $(\mathfrak{A}, \alpha)$ is said to be $\varphi$-supersymmetric if there exists a closable odd derivation $\widetilde{\delta}: \mathfrak{M}_{\mathrm{an}} \rightarrow \mathfrak{M}_{\mathrm{an}}$ such that

$$
\widetilde{\delta}^{2}(a)=\widetilde{\delta}_{0}(a), \quad a \in \mathfrak{M}_{\text {an }} .
$$


Here by an odd derivation we mean a graded derivation, i.e.,

$$
\widetilde{\delta}(a b)=\widetilde{\delta}(a) b+\widetilde{\gamma}(a) \widetilde{\delta}(b), \quad a, b \in \mathfrak{M}_{\mathrm{an}},
$$

which is odd, i.e., $\widetilde{\delta} \cdot \widetilde{\gamma}=-\widetilde{\gamma} \cdot \widetilde{\delta}$ on $\mathfrak{M}_{\text {an }}$. Note that the condition of $\varphi$-supersymmetry for a $\mathrm{C}^{*}$-dynamical system is somewhat weaker than the condition of supersymmetry since the existence of supersymmetry transformations is only required in the representation induced by $\varphi$.

Corollary 8. Let $\varphi$ be a graded KMS functional of the $\varphi$-supersymmetric $C^{*}$-dynamical system $(\mathfrak{A}, \alpha)$. If there exists a $\varphi$-asymptotically abelian sequence $\beta_{n} \in$ Aut $\mathfrak{A}$, then $\widetilde{\delta}=0, \widetilde{\alpha}=\mathrm{id}$, and $\widetilde{\varphi}$ is a trace.

Proof. By Proposition 7, $\widetilde{\varphi}$ is an ordinary KMS functional and the grading $\widetilde{\gamma}$ is trivial. Since $\widetilde{\delta}$ is odd, it follows that $\widetilde{\delta}=-\widetilde{\delta}=0$ on $\mathfrak{M}_{\text {an }}$, hence $\delta_{0}=0$ because of equation (19) and the fact that $\mathfrak{M}_{\text {an }}$ is a core for $\widetilde{\delta}_{0}$. Thus $\widetilde{\alpha}=\mathrm{id}$, hence by the KMS property we conclude that $\widetilde{\varphi}$ is a trace on $\mathfrak{M}$.

\section{Conclusions}

In the preceding analysis we have determined the form of graded KMS functionals of dynamical systems in a quite general setting and we want to discuss now some implications of our results for the study of infinite systems.

In order to fix ideas, let us assume that we are dealing with a family of graded dynamical systems $\left(\mathfrak{A}_{\Lambda}, \alpha_{\Lambda}, \gamma_{\Lambda}\right)$ which are assigned to compact subsets $\Lambda \subset \mathbb{R}^{n}$ and which determine in the limit $\Lambda \nearrow \mathbb{R}^{n}$ some dynamical system $(\mathfrak{A}, \alpha, \gamma)$ on which spatial translations act in an asymptotically abelian manner. This situation is familiar from quantum field theory, where one frequently constructs first the theory in a finite volume (box) $\Lambda$ and then proceeds to the thermodynamic limit.

For the finite volume theory one expects that the graded KMS functionals generically give rise to type I representations. They can then be presented in the familiar Gibbs form,

$$
\varphi_{\Lambda}(\cdot)=\operatorname{Tr} e^{-\beta H_{\Lambda}} V_{\Lambda} \cdot
$$


where $e^{-\beta H_{\Lambda}}$ is the density matrix of the Gibbs ensemble at inverse temperature $\beta$ and $V_{\Lambda}$ the unitary which implements the grading and commutes with the box-Hamiltonian $H_{\Lambda}$. If $\Lambda \nearrow \mathbb{R}^{n}$, the representation (21) is no longer meaningful, however, since the limit of $e^{-\beta H_{\Lambda}}$ is in general not a trace class operator. So the question arises in which sense a thermodynamic limit of graded Gibbs functionals can be defined.

From the point of view of physics it might seem natural to normalize the functionals, i.e., to proceed from $\varphi_{\Lambda}$ to $\left\|\varphi_{\Lambda}\right\|^{-1} \varphi_{\Lambda}$. The normalized functionals could then be interpreted as weighted differences of bosonic and fermionic ensembles [6] and the existence of (weak-*) limits would follow from standard compactness arguments. That this idea does not work in general can be seen if one thinks of the situation where one has a unique KMS state for given $\beta$, for example at high temperatures $\beta^{-1}$. Then the limit functionals are invariant under the spatial translations. But this is in conflict with Proposition 7 according to which the grading would have to be trivial, unless the limit is zero. So this approach does not seem viable.

The other obvious idea is to normalize the functionals by fixing the value of $\varphi_{\Lambda}(1)$ (the index), assuming that it is different from 0 . But then the norms $\left\|\varphi_{\Lambda}\right\|$ cannot stay bounded; for otherwise one would come, as above, to the conclusion that the limit points of the functionals $\varphi_{\Lambda}$ are zero in general, in conflict with their normalization. Hence in this approach one has to deal with unbounded sequences of functionals and it is not clear from the outset how a reasonable limit can be defined in these cases. To the best of our knowledge, this problem has not been solved so far in any non-trivial example.

From a mathematical point of view the second approach through unbounded functionals seems nevertheless attractive. For it provides a natural generalization of the concept of graded KMS functionals to the class of asymptotically abelian $\mathrm{C}^{*}$-dynamical systems. The unboundedness would not be an obstacle to the definition and analysis of entire cyclic cocyles [3] for such systems, should the functionals be continuous with respect to some auxiliary Banach algebra norm. It is less clear, however, how such unbounded functionals can be interpreted in physics. 


\section{Acknowledgements}

The second named author wishes to thank Klaus Fredenhagen for conversations. Both authors are grateful to the Erwin Schrödinger Institut in Vienna for hospitality and financial support, which made this collaboration possible.

\section{References}

[1] D. Buchholz and I. Ojima, Spontaneous collapse of supersymmetry, Nucl. Phys., B 498 (1997), 228.

[2] D. Buchholz, On the implementation of supersymmetry, in: Theoretical Physics Fin de Sieècle (Proceedings, Wroclaw 1998), A. Borowiecz et al. eds., Lect. Notes in Phys., 539, Springer 2000; hep-th 9812179.

[3] A. Connes, Noncommutative Geometry, Academic Press, 1994.

[4] J. Dixmier, C*-Algebras, North-Holland, 1977.

[5] J. Fuchs, Thermal and superthermal properties of supersymmetric field theories, Nucl. Phys., B 246 (1984), 279.

[6] L. van Hove, Supersymmetry and positive temperature for simple systems, Nucl. Phys., 207 (1982), 15.

[7] A. Jaffe, A. Lesniewski, and K. Osterwalder, Quantum K-theory, Commun. Math. Phys., 118 (1988), 1.

[8] D. Kastler, Cyclic cocycles from graded KMS functionals, Commun. Math. Phys., 121 (1989), 345.

[9] O. Stoytchev, The modular group and super-KMS functionals, Lett. Math. Phys., 27 (1993), 43.

[10] M. Takesaki, Tomita's Theory of Modular Hilbert Algebras, Lect. Notes in Math., 128, Springer, 1970.

[11] E. Witten, Constraints on supersymmetry breaking, Nucl. Phys., B 202 (1982), 253. 